\title{
Blood-brain barrier transport studies, aggregation, and molecular dynamics simulation of multiwalled carbon nanotube functionalized with fluorescein isothiocyanate
}

\author{
This article was published in the following Dove Press journal: \\ International Journal of Nanomedicine \\ 3 March 2015 \\ Number of times this article has been viewed
}

\author{
Sergey Shityakov' \\ Ellaine Salvador' \\ Giorgia Pastorin ${ }^{2}$ \\ Carola Förster' \\ 'Department of Anaesthesia and \\ Critical Care, University of Würzburg, \\ Würzburg, Germany; ${ }^{2}$ Department \\ of Pharmacy, National University \\ of Singapore, Singapore
}

\begin{abstract}
In this study, the ability of a multiwalled carbon nanotube functionalized with fluorescein isothiocyanate (MWCNT-FITC) was assessed as a prospective central nervous system-targeting drug delivery system to permeate the blood-brain barrier. The results indicated that the MWCNT-FITC conjugate is able to penetrate microvascular cerebral endothelial monolayers; its concentrations in the Transwell ${ }^{\circledR}$ system were fully equilibrated after 48 hours. Cell viability test, together with phase-contrast and fluorescence microscopies, did not detect any signs of MWCNT-FITC toxicity on the cerebral endothelial cells. These microscopic techniques also revealed presumably the intracellular localization of fluorescent MWCNT-FITCs apart from their massive nonfluorescent accumulation on the cellular surface due to nanotube lipophilic properties. In addition, the 1,000 ps molecular dynamics simulation in vacuo discovered the phenomenon of carbon nanotube aggregation driven by van der Waals forces via MWCNTFITC rapid dissociation as an intermediate phase.
\end{abstract}

Keywords: blood-brain barrier, multiwalled carbon nanotube, fluorescein isothiocyanate, Transwell ${ }^{\circledR}$ system, aggregation, fluorescence microscopy, molecular dynamics

\section{Introduction}

Targeted drug delivery across the blood-brain barrier (BBB) to the central nervous system (CNS) is a great challenge in the treatment of neurologic disorders. The existing and new biomedical approaches for CNS-active nanocarriers to improve drug delivery play an important role via enhancing their passive BBB permeation and active transport through the brain's endothelial cells. ${ }^{1}$ Therefore, the successful CNS-targeting drug-delivery vectors that cross the BBB without disruption have applications in many neurologic conditions, including Alzheimer's disease and multiple sclerosis. ${ }^{2,3}$ On the other hand, there is a significant misconception that small molecules can easily pass through the $\mathrm{BBB}$, but it has been affirmed that the majority of them do not permeate the BBB. ${ }^{4,5}$ Low molecular weight and high lipophilic properties are the factors that contribute to a better drug delivery in the $\mathrm{CNS}^{6}$

Carbon nanotubes (CNTs), with their rolled-up graphene structures, possess unique chemical, thermal, mechanical, and electrical properties, making them promising candidates for various biomedical applications. ${ }^{7,8}$ Functionalized CNTs as drug delivery vehicles to the CNS are receiving more attention from the scientific community. In particular, a dual-targeting drug delivery based on PEGylated oxidized multiwalled carbon nanotubes (MWCNTs) modified with angiopep-2 and single-walled carbon
Correspondence: Carola Förster; Sergey Shityakov

Department of Anesthesia and

Critical Care, University of Würzburg

Oberdürrbacher Street 6, 97080

Würzburg, Germany

Email foerster_c@ukw.de;

e_shityako_s@ukw.de
International Journal of Nanomedicine 2015:10 |703-17/3

1703

Dovepress

http://dx.doi.org// $0.2147 / 1 J N . S 68429$ License. The full terms of the License are available at http://creativecommons.org/licenses/by-nd/3.0/. Non-commercial uses of the work are permitted without any further (c) (i) (5) 2015 Shityakov et al. This work is published by Dove Medical Press Limited, and licensed under Creative Commons Attribution - Non Commercial (unported, v3.0) permission how to request permission may be found at: http://www.dovepress.com/permissions.php 
nanotube (SWCNT)-enhanced immunotherapy using $\mathrm{CpG}$ oligodeoxynucleotides were recently developed to inhibit tumor growth in glioma models. ${ }^{9,10}$

So far, very little has been known on the CNT uptake from the blood into the brain passing the BBB. It was previously reported that unmodified water-insoluble CNTs injected into the bloodstream of test animals were subsequently detected in small quantities in the brain tissue, indicating their ability to pass the BBB. ${ }^{11}$ Various studies have also described the nanotube biocompatibility effects that rat hippocampal neurons could grow along the MWCNT substrates, ${ }^{12}$ enhancing neural functional activity. ${ }^{13,14}$ However, the MWCNT incubation with cultured endothelial cells was linked to high levels of reactive oxygen species, proinflammatory cytokines, and cell adhesion molecules such as intracellular adhesion molecule 1 and vascular cell adhesion molecule $1 .^{15,16}$

Although the aggregation of pristine, non-functionalized MWCNTs in aqueous or organic phase imposes serious safety issues on their use and inhibits their widespread applications in pharmaceutical industry and neuroscience, ${ }^{17}$ this phenomenon still has not been well elucidated in respect to the BBB permeation for MWCNTs. The degree and type of MWCNT aggregation and accumulation are determined by the rigidity of nanotubes, and their diameters are thin enough to allow their buckling and self-aggregation into low-density, particle-like, intertwined, and coiled assemblies. ${ }^{18}$

In the current study, the MWCNT structures that consist of multiple concentric layers of carbon sheets and functionalized with fluorescein isothiocyanate (MWCNTFITC) as promising candidates of drug-delivery systems were evaluated for their permeability across microvascular cerebral endothelial (cEND) monolayers. Their accumulation and aggregation were also determined by phase-contrast and fluorescence microscopies in conjunction with molecular dynamics simulation.

\section{Materials and methods MWCNT-FITC synthesis}

Pristine MWCNTs purchased from Nanostructured and Amorphous Materials Inc. (Houston, TX, USA; Lot Number $1240 \mathrm{XH}$ ) were $94 \%$ pure, with an outer diameter of $37.39 \pm 12.62 \mathrm{~nm}$, length of $0.51 \pm 0.25 \mu \mathrm{m}$, and surface area of $0.001 \mu^{2}$. The MWCNT $-\mathrm{NH}_{3}{ }^{+}$intermediate was prepared as described in detail elsewhere. ${ }^{19-21}$ Fluorescent labeling of MWCNTs with fluorescein isocyanate was accomplished by reacting MWCNT $-\mathrm{NH}_{3}^{+}$with fluorescein isothiocyanate (FITC) in dimethylformamide and diisopropylethylamine at $60^{\circ} \mathrm{C}$ for 24 hours. $^{22}$

\section{Immunoblotting of tight junction-related proteins}

cEND cells were dissolved in Laemmli buffer ${ }^{23}$ with $5 \%$ $\beta$-mercaptoethanol and denatured at $95^{\circ} \mathrm{C}$ for 5 minutes. Proteins were subjected to sodium dodecyl sulfate-polyacrylamide gel electrophoresis (10\% SDS-PAGE gels) using a Mini TransBlot Electrophoretic Transfer Cell (Bio-Rad Laboratories, $\mathrm{GmbH}$, Munich, Germany) to wet-transfer them to a polyvinylidene difluoride membrane. The membrane was rinsed with Dulbecco's phosphate-buffered saline (PBS) (Sigma-Aldrich Chemie, GmbH, Hamburg, Germany), blocked in 5\% nonfat milk for 1 hour, and incubated overnight at $4^{\circ} \mathrm{C}$ in $\mathrm{PBS}$ with $0.5 \%$ bovine serum albumin (BSA) containing respective primary antibodies. The polyclonal antibodies against claudin-5, occludin, and $\beta$-actin were used at dilution rates of 1:400 (claudin-5 and occludin) and 1:2,500 ( $\beta$-actin), respectively. Subsequently, the membrane was washed three times for 15 minutes each with PBS and incubated at room temperature in 1\% BSA-PBS containing horseradish-peroxidase-labeled secondary antibody (Jackson ImmunoResearch Laboratories, Inc., West Grove, PA, USA). Bound immunoglobulins were visualized by the enhanced chemiluminescence method (GE Healthcare, Europe, GmbH, Freiburg, Germany). Densitometric analysis was performed with the MultiImage II imager and Fluorchem FC2 program (Biozym Scientific, GmbH, Hessisch Oldendorf, Germany). The ImageJ software (National Institute of Health, Bethesda, MD, USA) was used to analyze quantitatively the immunoblotting results.

\section{Transport studies and fluorescence microscopy}

Immortalized murine microvascular cEND cells ${ }^{24-26}$ were seeded on collagen type 4-coated Transwell ${ }^{\circledR}$ filters (Greiner Bio-One, GmbH, Frickenhausen, Germany) and cultured using Dulbecco's Modified Eagle's Medium (DMEM), $10 \%$ fetal calf serum (FCS), and penicillin/streptomycin mixture. The cells were grown for 5 days and differentiated with DMEM containing 1\% serum-stripped FCS to build up tight junctions for 2 days. Transendothelial electric resistance (TER) of the cell monolayer grown on the polyethylene terephthalate membrane with a pore diameter of $0.4 \mu \mathrm{m}$ was manually measured using the epithelial voltohmmeter device as a TER-monitoring system equipped with a fixed pair of STX2 electrodes, $4 \mathrm{~mm}$ wide and 1 mm thick (World Precision Instruments, Inc., Sarasota, FL, USA). Transendothelial resistances of blank filters, coated with collagen type 4, were used as controls and compared to those with cEND cells. MWCNTs functionalized with 
FITC in a concentration of $500 \mu \mathrm{mol} / \mathrm{g}$ were dispersed in PBS by sonication and carefully added to an apical chamber containing DMEM with $10 \%$ FCS in a final concentration of $40 \mu \mathrm{g} / \mathrm{mL}$. The measurements of FITC-labeled dextran 150 (150 kDa, FD150S, Sigma-Aldrich, St Louis, MO, USA) flux across monolayers of cultured cEND cells in the same concentration as for MWCNT-FITC were used as reliable control system widely implemented in various BBB transport experiments. ${ }^{27-29}$

FITC fluorescence was measured after 2, 24, and 48 hours by the Tecan GENios Microplate Reader (MTX Lab Systems, Inc., Vienna, VA, USA). Subsequently, the cEND cells were fixed with $2 \%$ of paraformaldehyde for 10-15 minutes and subjected to further analysis under the Olympus BX51 fluorescence microscope (Olympus Life Sciences Research Europa, GmbH, Munich, Germany), which was equipped with a digital camera to assess nanotube aggregation and accumulation. Pseudo-color fluorescent images were prepared, visualized, and analyzed with the Imaris v.7.2.3 image processing software (Bitplane, Inc., South Windsor, CT, USA). All experiments were repeated at least three times.

\section{Cell viability assay}

cEND cells were seeded in a 96-welled plate and grown to reach $90 \%$ of confluence in DMEM containing 10\% FCS, $50 \mathrm{U} / \mathrm{mL}$ penicillin/streptomycin, and 1\% L-glutamine. Once confluent, cells were forced to differentiate in $1 \%$ serumstripped FCS for 2 days. Next, cells were incubated with MWCNT-FITC in a final concentration of $40 \mu \mathrm{g} / \mathrm{mL}$ for 2,24 , and 48 hours at $37^{\circ} \mathrm{C}$. Afterward, cell viability was assessed using the CellTiter-Glo ${ }^{\circledR}$ luminescent cell viability assay (Promega, Madison, WI, USA) kit according to the manufacturer's instructions. Briefly, the test compound and controls were added to the cells, and after the intended incubation period, 30-minute incubation at room temperature followed. CellTiter-Glo ${ }^{\circledR}$ solution was then added. Lysis was induced for 2 minutes with shaking followed by a 10 -minute equilibration at room temperature. Luminescence was read using the Tecan GENios Microplate Reader (MTX Lab Systems, Inc.).

\section{Calculation of molecular descriptors}

The calculated octanol-water partitioning coefficient $(\mathrm{Clog} \mathrm{P})$, estimated permeability coefficient $\left(P_{\mathrm{e}}\right)$, absorption rate $\left(K_{\mathrm{a}}\right)$, plasma protein binding $(\% \mathrm{PPB})$, and human serum albumin affinity $\left(\log K_{\mathrm{a}}^{\mathrm{HSA}}\right)$ were determined by the ACD/I-Lab v.2.0 software with a proprietary algorithm v.5.0.0.184 (Advanced Chemistry Development, Inc., Toronto, ON, Canada) for
MWCNT-FITC as a measure of molecular hydrophobicity and pharmacokinetics. In addition, the topological polar surface area (TPSA), based on contributions of functional groups, was obtained using the Molecular Operating Environment v.2009.10 package (Chemical Computing Group, Montreal, QC, Canada).

\section{Molecular dynamics simulation and three-dimensional rendering}

The MWCNT structures were generated by Carbon Nanostructure Builder v.1.2 (Theoretical and Computational Biophysics Group, Beckman Institute, Urbana, IL, USA) with chiral indexes of 4 and 5, and $1.0 \mathrm{~nm}$ in length (Figure 1). The MarvinSketch software (ChemAxon, Ltd, Budapest, Hungary) was used to covalently attach FITC to the nanotube model. The molecular dynamics simulation was performed using the parameterized all-atom Merck molecular force field (MMFF94; Merck \& Co., Whitehouse Station, NJ, USA), which was previously reported as suitable for CNT molecular dynamics modeling. ${ }^{30}$ The energy minimization was initially performed using $0.001 \AA$ root mean square gradient. In the next step, the 1,000 ps molecular dynamics simulation in vacuo was performed with 2 fs time step using the NPT ensemble and NPA algorithm. The calculations and analyses were performed using the Molecular Operating Environment v.2009.10 (Chemical Computing Group) and VMD v.1.8.7 (Theoretical and Computational Biophysics Group) molecular modeling software suites.

The van der Waals (VDW) energies were computed with the Benchware ${ }^{\circledR}$ 3D Explorer program (Tripos International, St Louis, MO, USA). All nanotube rendering scenes and graphic representations were prepared with ambient occlusion lighting by the Tachyon ray tracer (Theoretical and Computational Biophysics Group), QuteMol v.0.4.1, ${ }^{31}$ MATLAB R2012a (MathWorks, Natick, MA, USA), and GraphPad Prism v.4 for Windows software (GraphPad Software, Inc., San Diego, CA, USA).

\section{Results and discussion}

Using a Transwell ${ }^{\circledR}$ model with $0.4 \mu \mathrm{m}$ pore size and $0.336 \mathrm{~cm}^{2}$ surface area of the filter membrane, the BBB permeation rate of MWCNT-FITC structure was accessed according to the previously described procedure (see Materials and methods section). Prior to and during this experiment, dynamic TER measurements of cEND monolayers with 2-, 24-, and 48-hour intervals were taken to validate the cellular tightness and provide paracellular property information. The cells exhibited TER values ranging from 


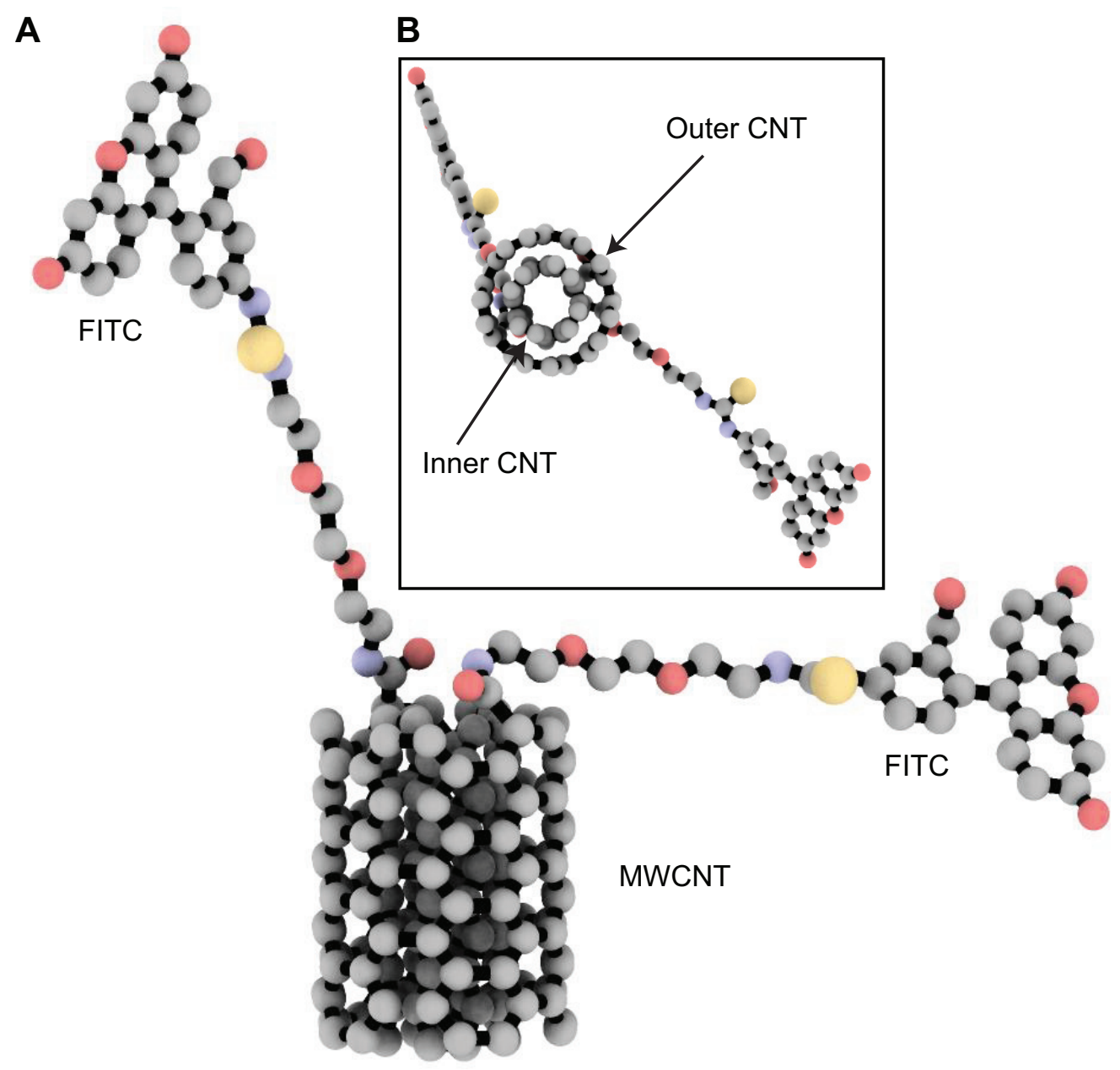

Figure I Three-dimensional molecular representation of the modeled MWCNT-FITC structure used in I,000 ps molecular dynamics simulation in vacuo. Notes: Frontal (A) and transverse (B) views. The molecule is rendered as ball-and-stick and coloured according to atom type without hydrogens to enhance clarity. Abbreviations: CNT, carbon nanotube; MWCNT-FITC, multiwalled carbon nanotube functionalized with fluorescein isothiocyanate.

$95.76 \pm 7.39(103.49 \pm 3.69)$ to $89.38 \pm 0.5(94.42 \pm 2.73) \Omega \mathrm{cm}^{2}$ after 2 and 48 hours of incubation with MWCNT-FITC (FITC-dextran) in comparison to the control group with the TER values ranging from $75.26 \pm 3.81$ to $69.88 \pm 2.02 \Omega \mathrm{cm}^{2}$ using empty inserts (Figure 2A). As depicted in Figure 2B, the BBB permeation of MWCNT-FITC from apical to basolateral chamber had started rapidly after 2 hours of incubation. Finally, the MWCNT-FITC system was fully equilibrated after 48 hours. On the contrary, the equilibration point for the FITC-labeled dextran 150 was not reached after 48 hours of incubation (Figure 2C). The BBB permeation of FITC-dextran system was severely obstructed because of the extensive branching behavior occurring at greater molecular weight $(\mathrm{MW}>10 \mathrm{kDa})^{32-34}$, high hydrophilic properties $(\log \mathrm{P}=-2.0$ for FITC-dextran $4 \mathrm{kDa}),{ }^{35}$ and topological polar surface area of dextrans $\left(268.68 \AA^{2}\right)$. To eliminate the possibility of FITC disintegration from dextran polymer, the FITC molecule was also covalently attached to dextran via random conjugation with any free hydroxyl group at a frequency of 0.003 to 0.02 moles of FITC per mole of glucose.

The BBB permeation propensity of MWCNT-FITC was also confirmed by its decimal logarithm of brainto-plasma concentration ratio ( $\log B \mathrm{~B})$ value of 0.536 calculated from modified Clark's equation ${ }^{36}$ : $\log \mathrm{BB}=0.152 \mathrm{Clog} \mathrm{P}-0.0148 \mathrm{TPSA}+0.139$, where $\mathrm{C} \log \mathrm{P}$ is the calculated octanol-water partitioning coefficient and TPSA is the topological polar surface area. In particular, since it was shown in many studies that chemical substances with $\log \mathrm{BB}>0(0.3)$ permeate the $\mathrm{BBB}$ readily, while molecules with $\log B \mathrm{~B}<0(-1.0)$ are usually poor BBB permeants, ${ }^{37-39}$ MWCNT-FITC can be attributed to the first group. Similarly, the estimated permeability coefficient $\left(P_{\mathrm{e}}=4.28 \times 10^{-4} \mathrm{~cm} \mathrm{~s}^{-1}\right)$ and absorption rate $\left(K_{\mathrm{a}}=0.029\right.$ $\mathrm{min}^{-1}$ ) at human jejunum scale ( $\mathrm{pH}$ 6.5) were predicted to be in favor of sufficient intestinal absorption for this type of chemical structure. 


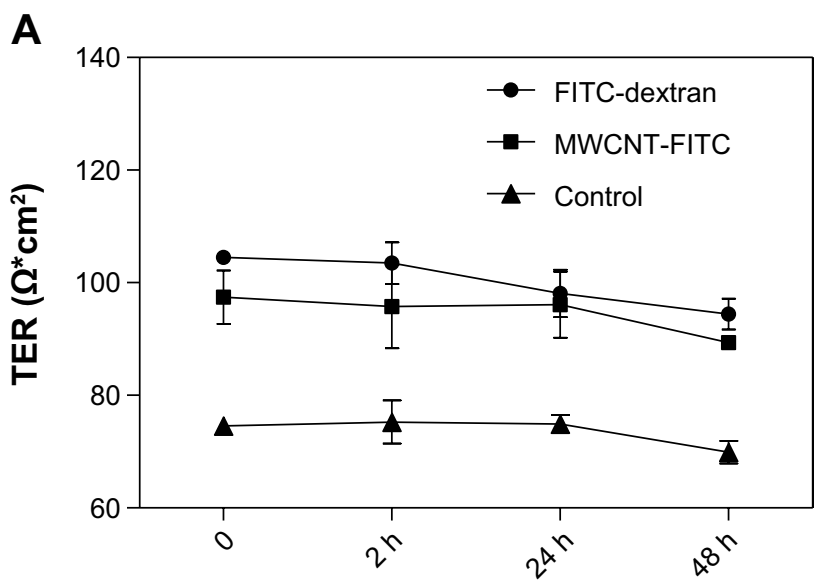

Time
B

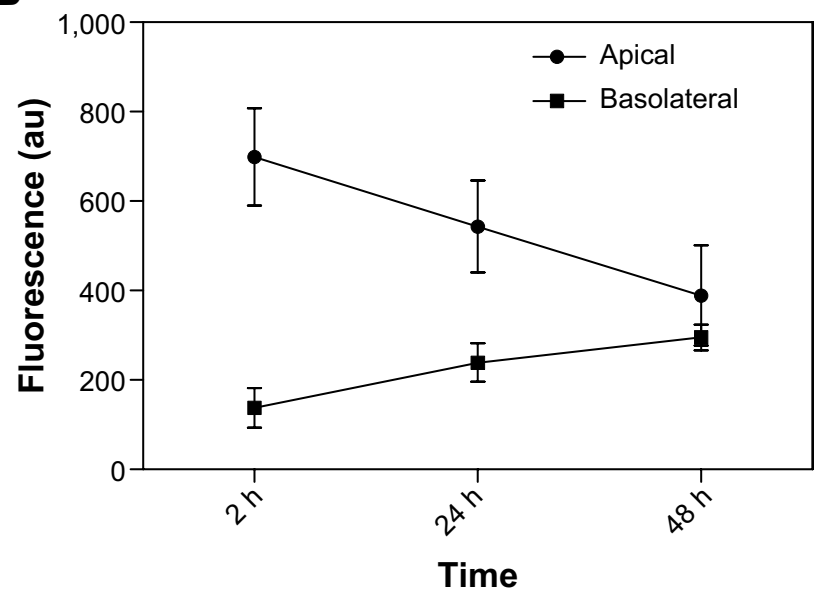

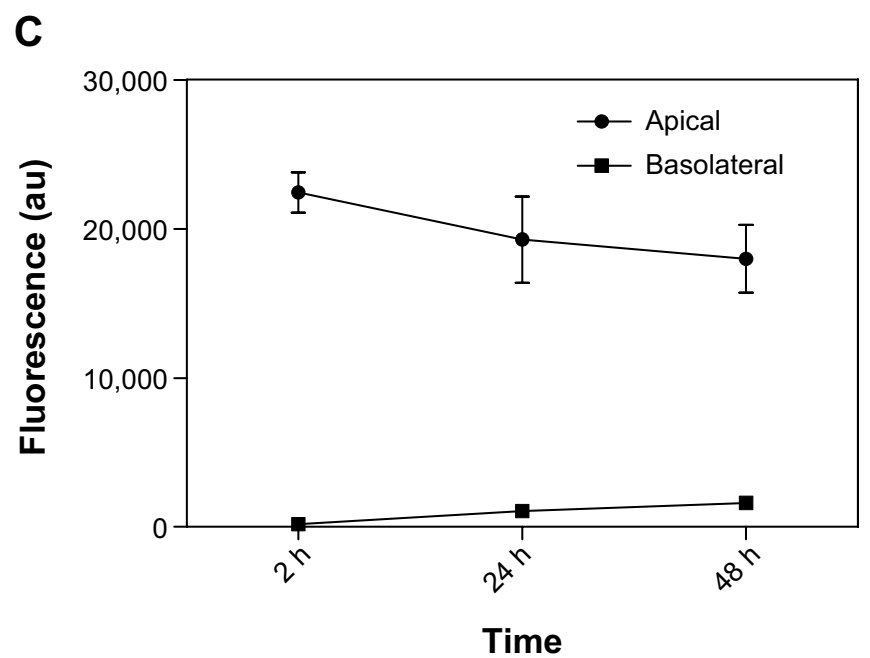

Figure 2 TER measurements of the cEND monolayers and the BBB transport studies of MWCNT-FITC and FITC-dextran.

Notes: Continuous recording of transendothelial electric resistance of the cEND monolayers seeded on collagen type 4-coated PET membranes in order to assess cell tightness after 2, 24, and 48 hours of MWCNT-FITC or FITC-dextran treatment (A). The BBB in vitro transport study of MWCNT-FITC (B) and FITC-dextran (C) for estimation of permeation rate. The fluorescence is measured in arbitrary units, abbreviated as au. Data represent means \pm SD of three independent experiments. Abbreviations: cEND, cerebral endothelial; PET, polyethylene terephthalate; MWCNT-FITC, multiwalled carbon nanotube functionalized with fluorescein isothiocyanate; FITC, fluorescein isothiocyanate; BBB, blood-brain barrier; TER, transendothelial electric resistance; SD, standard deviation; h, hours.

In the next step, the expression of tight junction proteins (occludin and claudin-5) in the cEND cells was analyzed by Western blotting technique in untreated control cEND cells and cells following 48 hours of MWCNT-FITC exposure in a concentration of $40 \mu \mathrm{g} / \mathrm{mL}$. As shown in Figure 3, the occludin and claudin-5 protein expression profiles at the steady state were the same in both control (untreated cultures) and MWCNT-treated cells. Although a slight elevation of tight junction proteins (ZO-1) has been previously reported by Rotoli et al investigating the impairment of barrier function in Calu-3 monolayers, caused by MWCNT structures, the change did not reach statistical significance because of the variability of absolute protein expression levels in the different experiments. ${ }^{40}$
The CellTiter-Glo ${ }^{\circledR}$ luminescent cell viability assay (Promega) was used to determine the cytotoxicity of MWCNTFITC. The results of this assay showed the absence of toxic effect for MWCNT-FITC on cEND cells after 2, 24, and 48 hours of incubation. Overall, with increased time, no significant difference from the actual cell viability either for MWCNT-FITC or for FITC-dextran was detected, and their luminescence levels remained above the $\mathrm{TD}_{50}$ (the median toxic dose) threshold. There was a significant cell death (significant reduction in luminescence activity) at $10 \%$ dimethyl sulfoxide, which served as a positive control (Figure 4).

Fluorescence microscopy was utilized to examine the MWCNT-FITC distribution, accumulation, and aggregation in the apical chamber containing DMEM with 10\% FCS. 
A

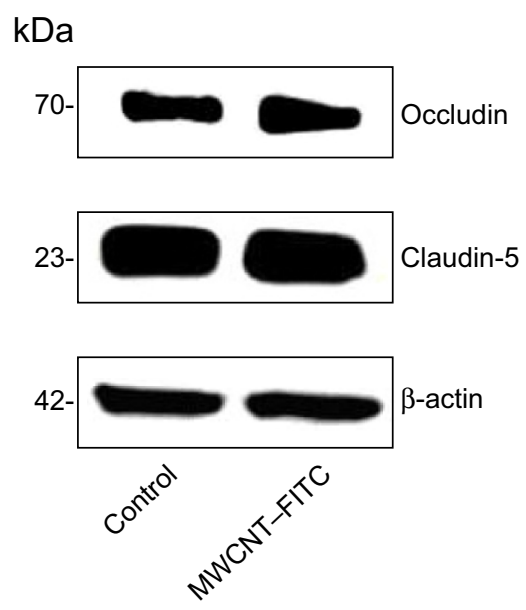

B

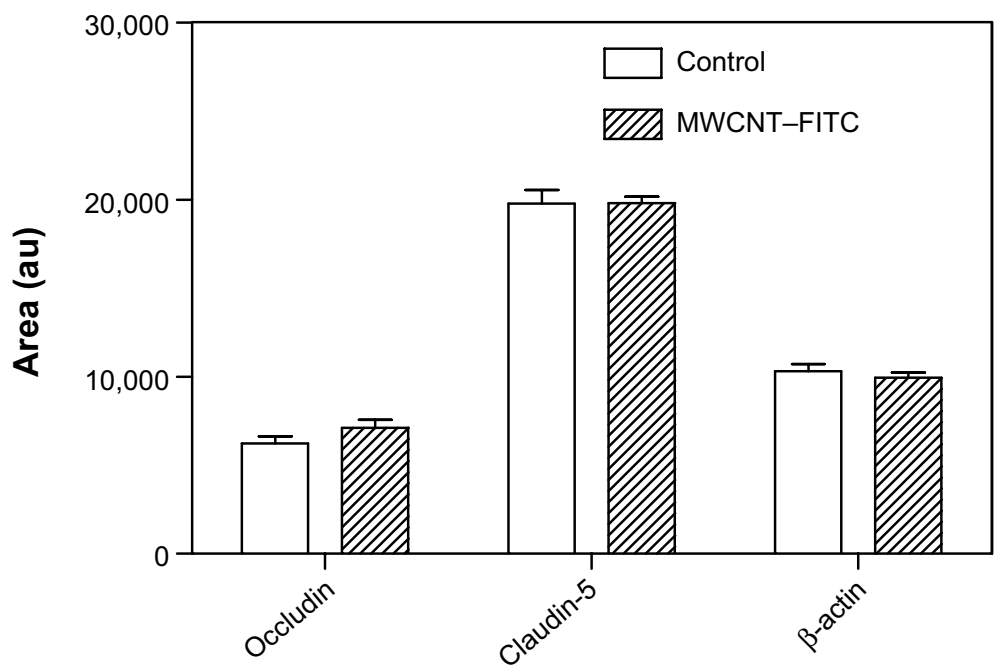

Figure 3 Western blot analysis of tight junction-related proteins expressed in cEND cells was performed using antibodies against occludin, claudin-5, and $\beta$-actin (internal/ loading control).

Notes: Expression profiles of tight junction-related proteins (occludin and claudin-5) in untreated (control) and MWCNT-FITC-treated cEND cells (A). Immunoblots analyzed and proteins quantified with the Image software (B). The area is measured in arbitrary units, abbreviated as au. Data represent means \pm SD of three independent experiments.

Abbreviations: MWCNT-FITC, multiwalled carbon nanotube functionalized with fluorescein isothiocyanate; cEND, cerebral endothelial; SD, standard deviation.

Figure 5A and B are fluorescent and phase-contrast micrographs, respectively, of the cEND cells on top of the polyethylene terephthalate membrane incubated at $37^{\circ} \mathrm{C}$ for 48 hours with MWCNT-FITC dispersions. Interestingly, the first observation was the lack of damaged monolayers and apoptotic cells, which might be associated with previously reported nontoxic effects of pristine and oxidized MWCNTs

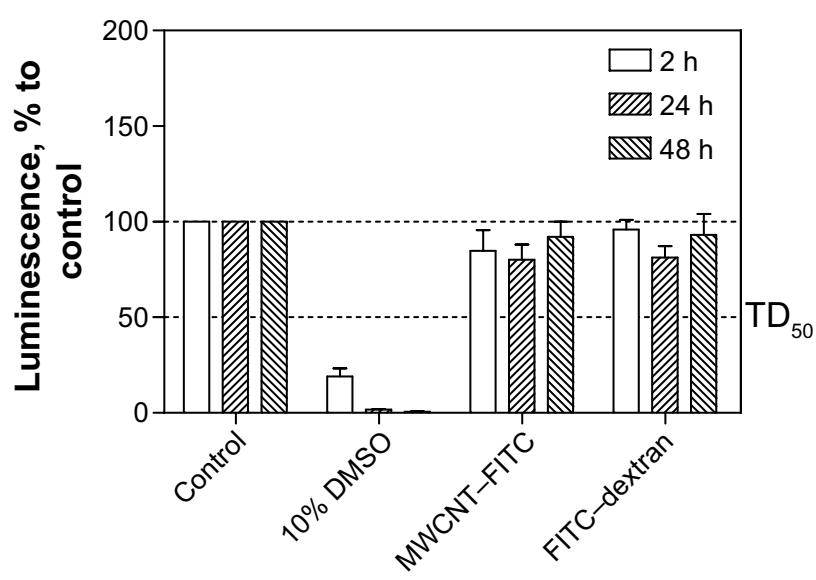

Figure 4 cEND cell viability to measure MWCNT-FITC toxic effect over time. Notes: Cell viability was assessed by the amount of ATP produced by metabolically active cells. The released ATP converts luciferin substrate to luciferin oxide, and released luminescence signals were recorded. The luminescence is measured in percentage to a control group (untreated cells). 10\% solution of DMSO was used as positive control. The thresholds are depicted as dashed lines. Data represent means \pm SD of three independent experiments.

Abbreviations: cEND, cerebral endothelial; MWCNT-FITC, multiwalled carbon nanotube functionalized with fluorescein isothiocyanate; ATP, adenosine triphosphate; DMSO, dimethyl sulfoxide; $\mathrm{TD}_{50}$, median toxic dose level; SD, standard deviation; h, hours. on the Caco-2 and RAW 264.7 mammalian cells using lactate dehydrogenase release assay. ${ }^{41}$

However, the most significant findings were the appearance of nonfluorescent black aggregated material most likely localized on the cell surface. It might be possible that when the aggregates are very dense and compact, they could "quench" the fluorescence. In contrast, the FITC-fluorescent patches of MWCNTs were presumably located in the cell interior or submerged in the lipid membrane because of endocytosis/phagocytosis or energyindependent passive processes as main transcellular routes of CNT internalization. ${ }^{42-44}$ Such observations are consistent with those of Kam et al who used confocal fluorescence microscopy to detect colocalization of fluorescently labeled nanotube conjugates. ${ }^{45}$ The agglomeration of functionalized MWCNTs was previously discovered around human ocular cells without significant changes in their morphology. ${ }^{46}$ As observed in Figure 5C and D, internalized MWCNTFITC conjugates were colocalized with blue-colored shapes of endothelial cells, with Pearson's coefficient of 0.915 indicating nanotube uptake without apparent adverse effects on cell viability.

The proper use of CNT vectors for targeted drug delivery is directly connected with overcoming aggregation and agglomeration problems. Moreover, these chemical structures behave as a weak base (base $\mathrm{p} K_{\mathrm{a}}<8.5$ ) and predominantly bind to human serum albumin and $\alpha 1$-acid glycoprotein $\left(\% \mathrm{PPB}=99.9 \%\right.$ and $\left.\log K_{\mathrm{a}}^{\mathrm{HSA}}=23.72\right)$, 

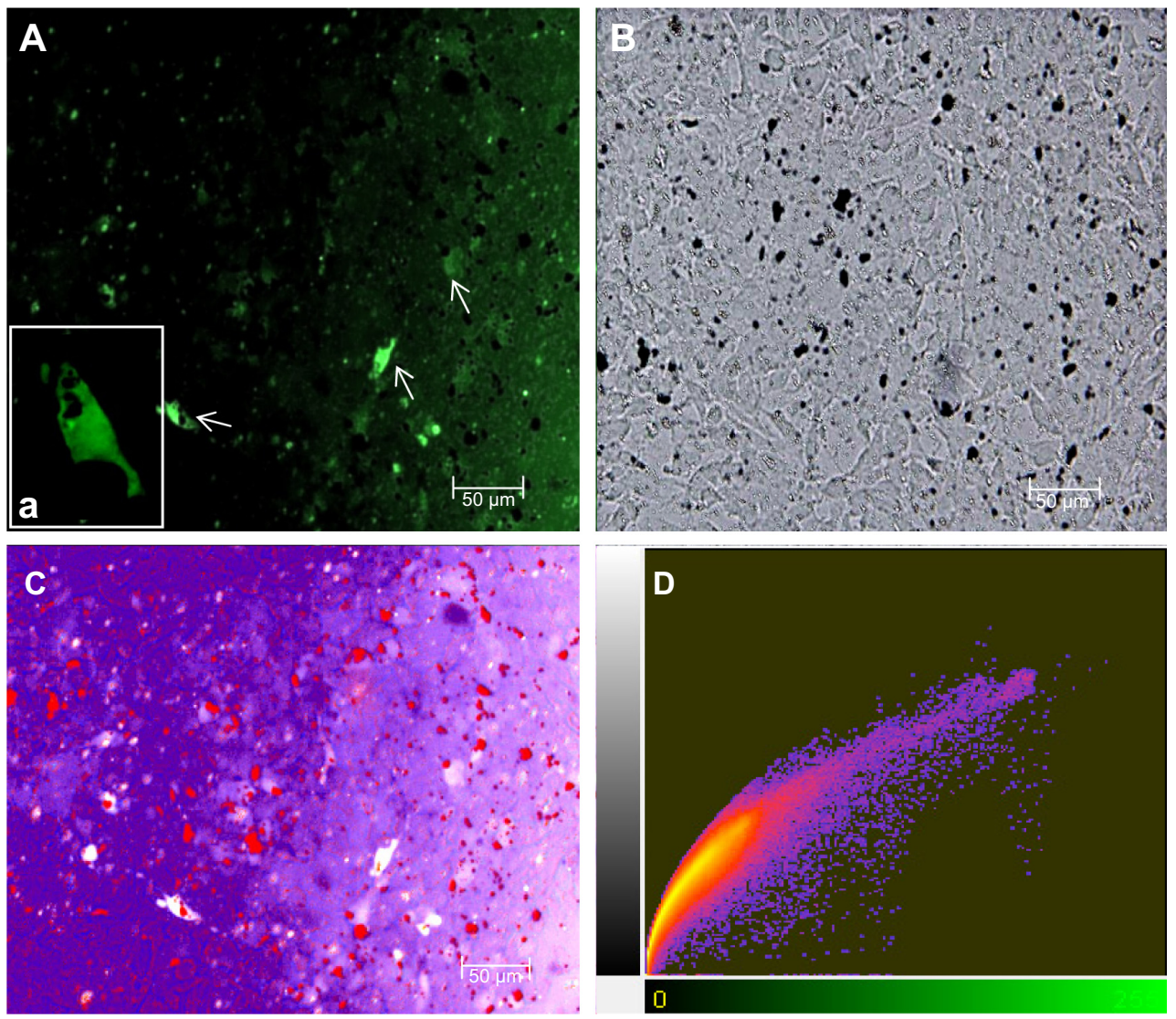

Figure 5 Fluorescent and phase-contrast microscopy with image analysis of the cEND cell monolayers treated with FITC-conjugated MWCNTs.

Notes: Low (A) and high (a) magnification (scale bar $=25 \mu \mathrm{m}$ ) fluorescent microscopic images together with conventional light (phase-contrast) (B) microscopy are shown for the cEND cell monolayers treated with FITC-conjugated MWCNTs, which were accumulated in cells (shown with arrows). Pseudo-colour fluorescent image (C) and correlation plot (D) to identify the amount of signal colocalization and intensity distribution. Cells are blue, nanotube aggregates are red, and colocalized spots are white.

Abbreviations: cEND, cerebral endothelial; FITC, fluorescein isothiocyanate; MWCNT, multiwalled carbon nanotube.

which might enhance CNT aggregation in the blood. Although, MWCNT-FITC aggregates were dispersed via sonication, they still had the tendency to reappear as a part of secondary aggregation. Different MWCNT-FITC aggregation types (I-IV) consisting of nanotube "bundles" were detected, and their ability to penetrate the polyethylene terephthalate membrane pore with a diameter of $0.4 \mu \mathrm{m}$ and a circle surface area of $0.13 \mu \mathrm{m}^{2}$ was further investigated by comparing their geometric properties (Table 1). Regardless of the high lipophilic characteristics $(\mathrm{C} \log \mathrm{P}=30.16)$ of the nanotubes, the pore's sphere surface area $\left(0.5 \mu \mathrm{m}^{2}\right)$ and volume $\left(0.03 \mu \mathrm{m}^{3}\right)$ were the main factors blocking the BBB permeation of bulky MWCNT-FITC aggregates (Table 2).

In order to reconstruct a three-dimensional (3D) image of bulky MWCNT-FITC aggregates, a 3D intensity plot was generated from the two-dimensional three-colored image via a MATLAB script using meshing techniques with color separation and axial interactive rotation. To represent 3D data correctly, a third dimension was adjusted with respect to the color intensity gradation map to make the minimum height correspond to the cEND cells and the maximum to the large nanotube aggregates. This 3D aggregate reconstruction aimed to provide some additional information with respect to the approximate MWCNT-FITC aggregation size and shape, revealing and emphasizing its extracellular localization and level of distribution (Figure 6).

Finally, the 1,000 ps molecular dynamics simulation in vacuo of eight MWCNT-FITC molecules provided additional information on CNT aggregation mechanisms at the molecular level following the rapid dissociation of their inner and outer parts during an intermediate phase after 1-5 ps. The absence of net charge on CNTs was due to the nonionized state of the molecule with no acid groups (with $\mathrm{p} K_{\mathrm{a}}<4.5$ ) and no base groups (with $\mathrm{p} K_{\mathrm{a}} \geq 7.0$ ) present. Therefore, the VDW interaction was the only driving force to run the aggregation mechanism. As a result, three types of nanotube aggregates were clearly detected even after the first $100 \mathrm{ps}$ of simulation: SWCNT dimer, SWCNT-FITC dimer, and 
Table I Summary of main geometric properties for different MWCNT-FITC aggregation types (I-IV) from the in vitro BBB transport study

\begin{tabular}{llllll}
\hline Type & Area $\left(\mu \mathrm{m}^{2}\right)$ & Count & Average diameter $(\mu \mathrm{m})$ & Total area $\left(\mu \mathrm{m}^{2}\right)$ & Area fraction $(\%)$ \\
\hline I & $>100$ & $\mathrm{I}$ & 11.982 & 112.774 & 0.08 \\
II & $50-100$ & 4 & 9.143 & 262.648 & 0.187 \\
III & $1.0-50$ & 171 & $3.4 I I$ & $1,562.769$ & 1.114 \\
IV & $0.13-1.0$ & 183 & 0.739 & 78.69 & 0.056 \\
I-IV & $0.13-100$ & 359 & 2.602 & $1,909.162$ & 1.357 \\
\hline
\end{tabular}

Abbreviations: MWCNT-FITC, multiwalled carbon nanotube functionalized with fluorescein isothiocyanate; BBB, blood-brain barrier.

SWCNT/SWCNT-FITC hybrid pentamer (Figure 7). In rough approximation, the entire mechanism of the MWCNTFITC aggregation could be depicted as a formation of these aggregates via the initial MWCNT-FITC disintegration into SWCNT and SWCNT-FITC, where the VDW energies of isolated SWCNT (17.72 kcal mol-1) and SWCNT-FITC $\left(-0.177 \mathrm{kcal} \mathrm{mol}^{-1}\right)$ were taken separately as the "initial state" energy. Considering a VDW-dependent aggregation mechanism, the VDW aggregation energy $\left(E_{\mathrm{VDW}}^{\text {agregation }}\right)$ was calculated from the following equation (Table 3 ):

$$
E_{\mathrm{VDW}}^{\text {agregation }}=E_{\mathrm{VDW}}^{\text {aggregate }}-\sum_{E_{\mathrm{VDW}}^{\mathrm{comp}}=1}^{\infty} E_{\mathrm{VDW}}^{\mathrm{comp}} \text {, }
$$

where $E_{\mathrm{vDW}}^{\text {aggregate }}$ is the total VDW energy of CNT aggregate, and $\sum_{E_{\mathrm{VDW}}^{\text {comp }}=1}^{\infty} E_{\mathrm{VDW}}^{\mathrm{comp}}$ is the sum of initial-state VDW energies of the components forming an aggregated nanoparticle.

It is also important to note that the VDW energy for SWCNT was found to be positive. A possible explanation might be that at the beginning of the molecular dynamics simulation, the MWCNT-FITC structure experienced a strong repulsion effect between its inner and outer parts because of the overlapping of their electron clouds. In contrast, the $E_{\mathrm{VDW}}^{\mathrm{SWCNT}-\mathrm{FITC}}$ parameter has a negative value, favoring molecular attraction with a strong tendency to orient nanotubes parallel to each other in the form of ropes and bundles.
Previous studies have shown this nanotube self-assembly and agglomeration to be dependent on the type of chemical functionalization (usually through strong acid oxidation) and surface-active dispersing agents, such as surfactants. ${ }^{47,48}$

\section{Conclusion}

Overall, the ability of an MWCNT-FITC as a prospective CNS-targeting drug delivery vector to permeate the BBB was investigated. In our previous study, we found evidence that at least $30 \%-50 \%$ of chemically functionalized MWCNTs were taken up by cells through an energy-independent passive mechanism. ${ }^{49}$ This characteristic makes nanotubes loaded with therapeutic or diagnostic cargos extremely interesting, as the release of active molecules directly into the cytoplasm increases their biological activity and therapeutic efficacy. The results obtained from this study indicated that the FITCfunctionalized MWCNTs were able to penetrate the cEND monolayers, reaching full equilibration in the Transwell ${ }^{\circledR}$ system after 48 hours. Phase-contrast and fluorescence microscopies determined intact cEND monolayers after a 48-hour exposure of MWCNT-FITC. Regardless of the fact that the massive CNT aggregation was observed as nonfluorescent clusters which were most likely located on the surface of the cEND cells, the toxic effects of MWCNTFITC on cellular viability were not detected. In conclusion, the 1,000 ps molecular dynamics simulation in vacuo confirmed the hypothesis that these nanotubes aggregate through the total decrease of VDW energy to occupy the lowest

Table 2 Summary of average geometric characteristics for different MWCNT-FITC aggregation types (I-IV) from the in vitro BBB transport study

\begin{tabular}{llll}
\hline Type & $\begin{array}{l}\text { Average surface } \\
\text { area }\left(\mu \mathrm{m}^{2}\right)\end{array}$ & $\begin{array}{l}\text { Average sphere } \\
\text { surface area }\left(\mu \mathrm{m}^{2}\right)\end{array}$ & $\begin{array}{l}\text { Average } \\
\text { volume }\left(\mu \mathrm{m}^{3}\right)\end{array}$ \\
\hline I & II 2.774 & 451.096 & 900.901 \\
II & 65.662 & 262.648 & 400.253 \\
III & 9.139 & 36.555 & 20.783 \\
IV & 0.430 & 1.72 & 0.212 \\
I-IV & 5.318 & 21.272 & 9.225 \\
\hline
\end{tabular}

Abbreviations: MWCNT-FITC, multiwalled carbon nanotube functionalized with fluorescein isothiocyanate; BBB, blood-brain barrier. 


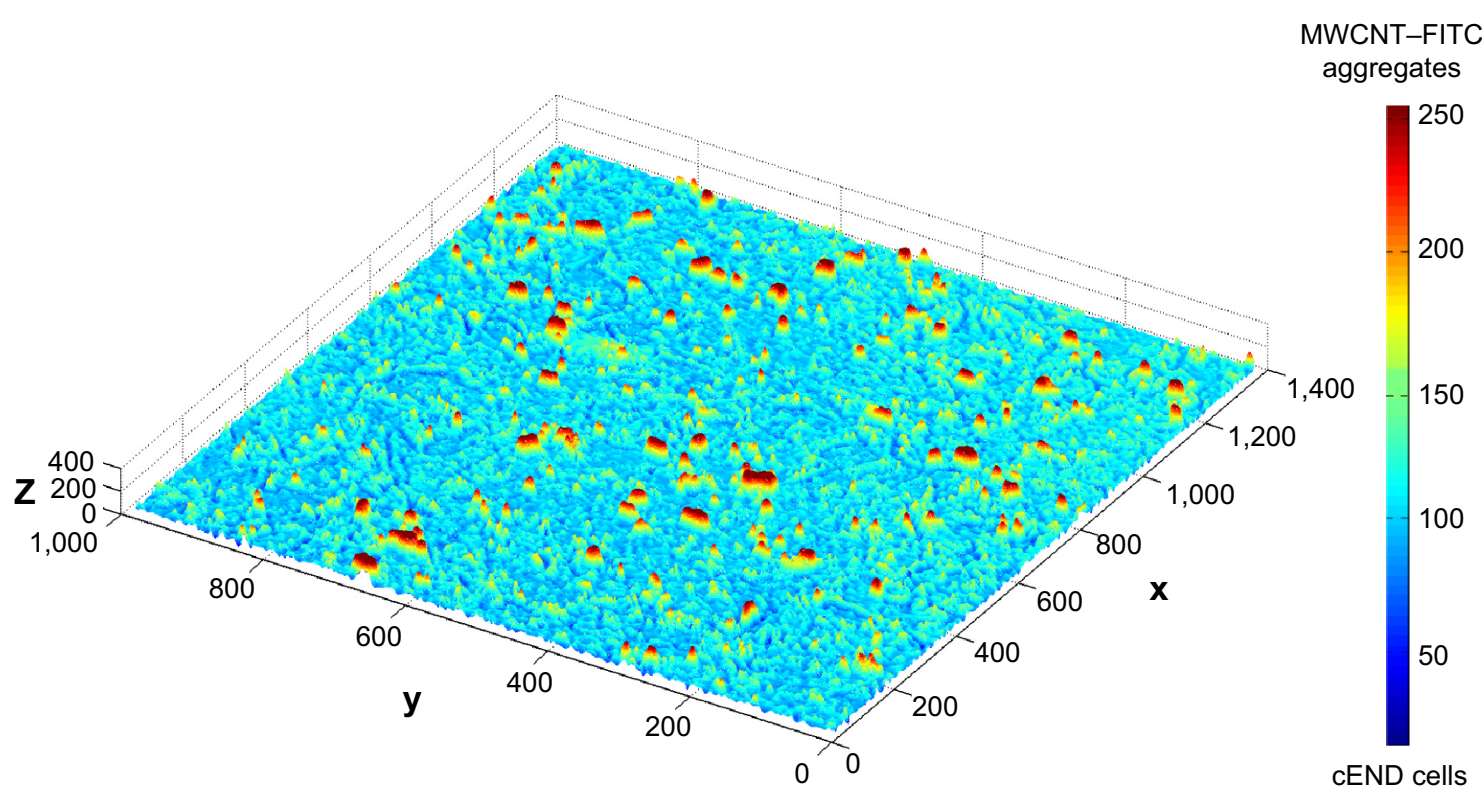

Figure 6 Three-dimensional mesh intensity plot of the bulky MWCNT-FITC aggregates (red) on PET membrane covered with confluent cEND cells (blue). Abbreviations: cEND, cerebral endothelial; MWCNT-FITC, multiwalled carbon nanotube functionalized with fluorescein isothiocyanate.

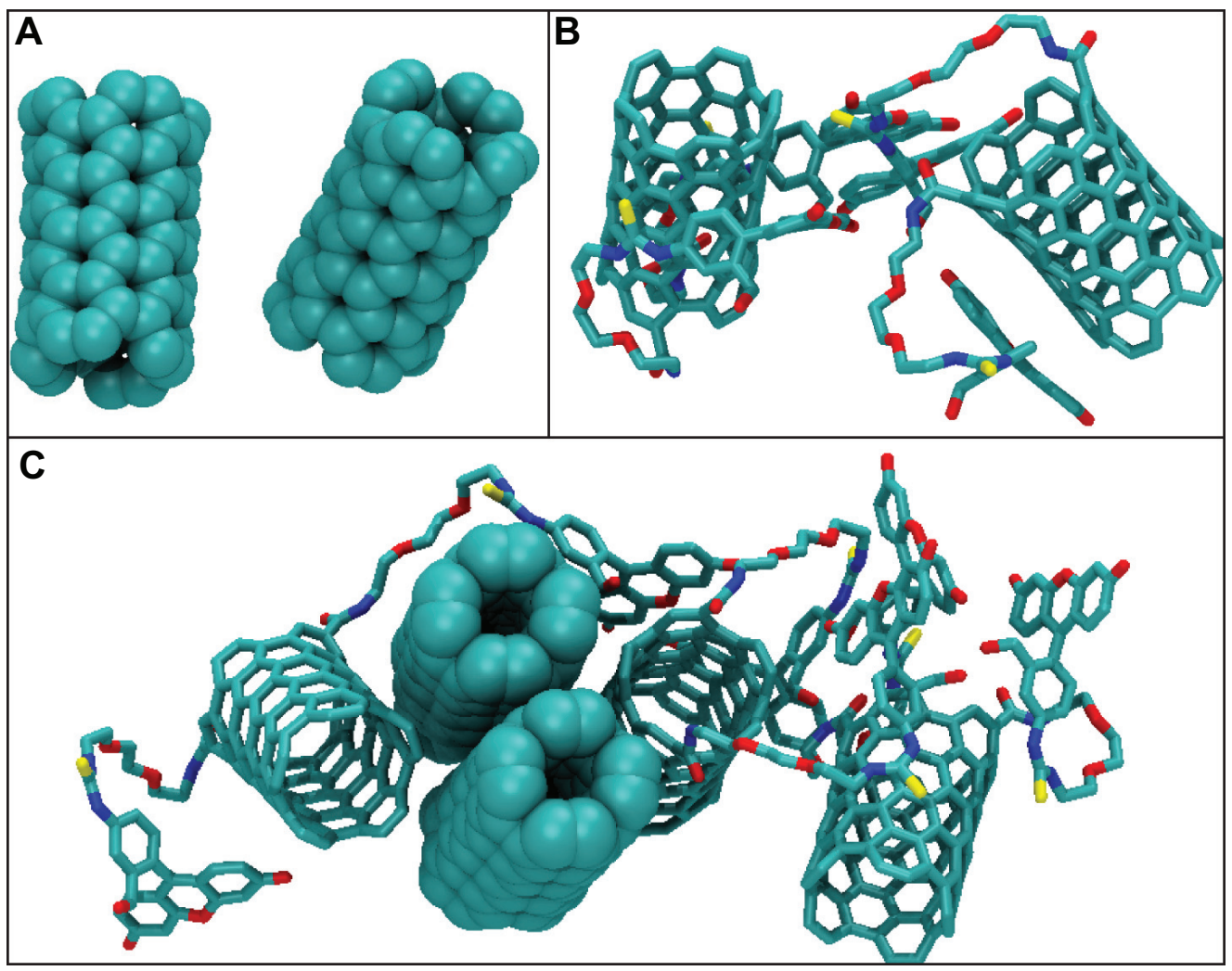

Figure 7 All-atom molecular dynamics simulation of MWCNT-FITC structures in vacuo.

Notes: Visualization of the carbon nanotube aggregates determined from I,000 ps molecular dynamics simulation of MWCNT-FITC: the SWCNT (A) and SWCNT-FITC (B) dimers, and SWCNT/SWCNT-FITC hybrid pentamer (C). These nanotube aggregates are represented as space-filling diagram and licorice models coloured according to their atomic composition. Hydrogen atoms are omitted for clarity.

Abbreviations: MWCNT-FITC, multiwalled carbon nanotube functionalized with fluorescein isothiocyanate; SWCNT, single-walled carbon nanotube; SWCNT-FITC, single-walled carbon nanotube functionalized with fluorescein isothiocyanate. 
Table 3 Summary of VDW aggregation energy for different carbon nanotube aggregates determined from I,000 ps molecular dynamics study of MWCNT-FITC

\begin{tabular}{lll}
\hline Aggregate & $E_{\text {VDW }}^{\text {aggregate }}\left(\mathrm{kcal} \mathrm{mol}^{-1}\right)$ & $E_{\text {VDW }}^{\text {aggregation }}\left(\mathrm{kcal} \mathrm{mol}^{-1}\right)$ \\
\hline SWCNT dimer & 26.5 & -8.94 \\
SWCNT-FITC dimer & -67 & -66.65 \\
Hybrid pentamer & -52 & -86.91 \\
\hline
\end{tabular}

Abbreviations: VDW, van der Waals; MWCNT-FITC, multiwalled carbon nanotube functionalized with fluorescein isothiocyanate; SWCNT, single-walled carbon nanotube; SWCNT-FITC, single-walled carbon nanotube functionalized with fluorescein isothiocyanate.

energy levels. This phenomenon was driven mainly by the VDW forces via MWCNT-FITC rapid dissociation as an intermediate phase. Therefore, it is important to improve this promising nano-vector for successful CNS-targeting therapy of various neurological disorders by decreasing its aggregation and increasing its BBB permeation potential.

\section{Acknowledgments}

Special thanks are extended to Anna Poon from the City College of New York for her assistance in the writing of this paper. The authors are also grateful to the BMBF (Bundesministerium für Bildung und Forschung) for their support of this work by providing the BMBF01 grant to Carola Förster.

\section{Disclosure}

The authors report no conflicts of interest in this work.

\section{References}

1. Béduneau A, Saulnier P, Benoit JP. Active targeting of brain tumors using nanocarriers. Biomaterials. 2007;28(33):4947-4967.

2. Li HY, Luo Y, Derreumax P, Wei G. Carbon nanotube inhibits the formation of beta-sheet-rich oligomers of the Alzheimer's amyloid-beta(16-22) peptide. Biophys J. 2011;101(9):2267-2276.

3. Ionescu R, Broza Y, Shaltieli H, et al. Detection of multiple sclerosis from exhaled breath using bilayers of polycyclic aromatic hydrocarbons and single-wall carbon nanotubes. ACS Chem Neurosci. 2011;2(12):687-693.

4. Pardridge WM. The blood-brain barrier: bottleneck in brain drug development. Neuro Rx. 2005;2(1):3-14.

5. Shityakov S, Salvador E, Förster C. In silico, in vitro and in vivo methods to analyse drug permeation across the blood-brain barrier: a critical review. OA Anaesth. 2013;1(2):13.

6. Pardridge WM. Brain Drug Targeting: the Future of Brain Drug Development. Cambridge, UK: Cambridge University Press; 2001.

7. Hu WH, Lu ZS, Liu Y, Li CM. In situ surface plasmon resonance investigation of the assembly process of multi-walled carbon nanotubes on an alkanethiol self-assembled monolayer for efficient protein immobilization and detection. Langmuir. 2010;26(11):8386-8391.

8. Wang WZ, Li WF, Pan XY, et al. Degradable conjugated polymers: synthesis and applications in enrichment of semiconducting single-walled carbon nanotubes. Adv Funct Mater. 2011;21(9):1643-1651.

9. Zhao D, Alizadeh D, Zhang L, et al. Carbon nanotubes enhance CpG uptake and potentiate antiglioma immunity. Clin Cancer Res. 2011;17(4): 771-782.
10. Ren J, Shen S, Wang D, et al. The targeted delivery of anticancer drugs to brain glioma by PEGylated oxidized multi-walled carbon nanotubes modified with angiopep-2. Biomaterials. 2012;33(11):3324-3333.

11. Yang ST, Guo W, Lin Y, et al. Biodistribution of pristine single-walled carbon nanotubes in vivo. $J$ Phys Chem C. 2007;111(48):17761-17764.

12. Mattson MP, Haddon RC, Rao AM. Molecular functionalization of carbon nanotubes and use as substrates for neuronal growth. $J \mathrm{Mol}$ Neurosci. 2000;14(3):175-182.

13. Lovat V, Pantarotto D, Lagostena L, et al. Carbon nanotube substrates boost neuronal electrical signaling. Nano Lett. 2005;5(6):1107-1110.

14. Matsumoto K, Sato C, Naka Y, Kitazawa A, Whitby RL, Shimizu N. Neurite outgrowths of neurons with neurotrophin-coated carbon nanotubes. J Biosci Bioeng. 2007;103(3):216-220.

15. Vidanapathirana AK, Lai XY, Hilderbrand SC, et al. Multi-walled carbon nanotube directed gene and protein expression in cultured human aortic endothelial cells is influenced by suspension medium. Toxicology. 2012;302(2-3):114-122.

16. Cao Y, Jacobsen NR, Danielsen PH, et al. Vascular effects of multiwalled carbon nanotubes in dyslipidemic apoE(/) mice and cultured endothelial cells. Toxicol Sci. 2014;138(1):104-116.

17. Jiang LQ, Gao L, Sun J. Production of aqueous colloidal dispersions of carbon nanotubes." J Colloid Interface Sci. 2003;260(1):89-94.

18. Pauluhn J. Subchronic 13-week inhalation exposure of rats to multiwalled carbon nanotubes: toxic effects are determined by density of agglomerate structures, not fibrillar structures. Toxicol Sci. 2010;113(1): 226-242.

19. Georgakilas V, Kordatos K, Prato M, Guldi DM, Holzinger M, Hirsch A. Organic functionalization of carbon nanotubes. $J$ Am Chem Soc. 2002;124(5):760-761.

20. Gaillard C, Cellot G, Li S, et al. Carbon nanotubes carrying cell-adhesion peptides do not interfere with neuronal functionality. Adv Mater. 2009; 21(28):2903-2908

21. Serag MF, Kaji N, Gaillard C, et al. Trafficking and subcellular localization of multi-walled carbon nanotubes in plant cells. ACS Nano. 2011;5(1):493-499.

22. Yoong SL, Wong BS, Zhou QL, et al. Enhanced cytotoxicity to cancer cells by mitochondria-targeting MWCNTs containing platinum (IV) prodrug of cisplatin. Biomaterials. 2014;35(2):748-759.

23. Laemmli UK. Cleavage of structural proteins during the assembly of the head of bacteriophage T4. Nature. 1970;227(5259):680-685.

24. Förster C, Silwedel C, Golenhofen N, et al. Occludin as direct target for glucocorticoid-induced improvement of blood-brain barrier properties in a murine in vitro system. $J$ Physiol. 2005;565(pt 2):475-486.

25. Silwedel C, Forster C. Differential susceptibility of cerebral and cerebellar murine brain microvascular endothelial cells to loss of barrier properties in response to inflammatory stimuli. J Neuroimmunol. 2006; 179(1-2):37-45.

26. Golenhofen N, Ness W, Wawrousek EF, Drenckhahn D. Expression and induction of the stress protein alpha-B-crystallin in vascular endothelial cells. Histochem Cell Biol. 2002;117(3):203-209.

27. Schreibelt G, Musters RJ, Reijerkerk A, et al. Lipoic acid affects cellular migration into the central nervous system and stabilizes blood-brain barrier integrity. J Immunol. 2006;177(4):2630-2637.

28. Fabis MJ, Phares TW, Kean RB, Koprowski H, Hooper DC. Blood-brain barrier changes and cell invasion differ between therapeutic immune clearance of neurotrophic virus and CNS autoimmunity. Proc Natl Acad Sci US A. 2008;105(40):15511-15516.

29. Chen F, Radisky ES, Das P, et al. TIMP-1 attenuates blood-brain barrier permeability in mice with acute liver failure." J Cereb Blood Flow Metab. 2013;33(7):1041-1049.

30. Shityakov S, Dandekar T. Molecular dynamics simulation of popc and pope lipid membrane bilayers enforced by an intercalated single-wall carbon nanotube. Nano. 2011;6(1):19-29.

31. Tarini M, Cignoni P, Montani C. Ambient occlusion and edge cueing to enhance real time molecular visualization. IEEE Trans Vis Comput Graph. 2006;12(5):1237-1244. 
32. Senti FR, Hellman NN, Ludwig NH, et al. Viscosity, sedimentation, and light-scattering properties of fraction of an acid-hydrolyzed dextran. $J$ Polym Sci. 1955;17(86):527-546.

33. Granath KA. Solution properties of branched dextrans. J Colloid Sci. 1958;13(4):308-328.

34. Basedow AM, Ebert KH. Production, characterization, and solution properties of dextran fractions of narrow molecular weight distributions. J Polym Sci. 1979;66(1):101-115.

35. Sakai M, Imai T, Ohtake H, Azuma H, Otagiri M. Effects of absorption enhancers on the transport of model compounds in Caco-2 cell monolayers: assessment by confocal laser scanning microscopy. J Pharm Sci. 1997;86(7):779-785.

36. Clark DE. Rapid calculation of polar molecular surface area and its application to the prediction of transport phenomena. 2. Prediction of blood-brain barrier penetration. J Pharm Sci. 1999;88(8):815-821.

37. Abraham MH, Takacs-Novak K, Mitchell RC. On the partition of ampholytes: application to blood-brain distribution. J Pharm Sci. 1997; 86(3):310-315.

38. Vilar S, Chakrabarti M, Costanzi S. Prediction of passive blood-brain partitioning: straightforward and effective classification models based on in silico derived physicochemical descriptors. J Mol Graph Model. 2010;28(8):899-903

39. Shityakov S, Broscheit J, Förster C. alpha-Cyclodextrin dimer complexes of dopamine and levodopa derivatives to assess drug delivery to the central nervous system: ADME and molecular docking studies. Int J Nanomed. 2012;7:3211-3219.

40. Rotoli BM, Bussolati O, Barilli A, et al. Airway barrier dysfunction induced by exposure to carbon nanotubes in vitro: which role for fiber length? Hum Exp Toxicol. 2009;28(6-7):361-368.

41. Clark KA, O’Driscoll C, Cooke CA, et al. Evaluation of the interactions between multiwalled carbon nanotubes and Caco-2 cells. J Toxicol Environ Health A. 2012;75(1):25-35.
42. Cherukuri P, Bachilo SM, Litovsky SH, Weisman RB. Near-infrared fluorescence microscopy of single-walled carbon nanotubes in phagocytic cells. J Am Chem Soc. 2004;126(48):15638-15639.

43. Singh R, Pantarotto D, McCarthy D, et al. Binding and condensation of plasmid DNA onto functionalized carbon nanotubes: toward the construction of nanotube-based gene delivery vectors. J Am Chem Soc. 2005; 127(12):4388-4396

44. Firme CP 3rd, Bandaru PR. Toxicity issues in the application of carbon nanotubes to biological systems. Nanomedicine. 2010;6(2):245-256.

45. Kam NWS, Jessop TC, et al. Nanotube molecular transporters Internalization of carbon nanotube-protein conjugates into mammalian cells. J Am Chem Soc. 2004;126(22):6850-6851.

46. Yan L, Li GX, Shu Z, et al. Cytotoxicity and genotoxicity of multiwalled carbon nanotubes with human ocular cells. Chin Sci Bull. 2013 58(19):2347-2352.

47. Fraczek-Szczypta A, Menaszek E, et al. Some observations on carbon nanotubes susceptibility to cell phagocytosis. J Nanomater. 2011;2011, Article ID 473516.

48. Bouchard D, Zhang W, Powell T, Rattanaudompol US. Aggregation kinetics and transport of single-walled carbon nanotubes at low surfactant concentrations. Environ Sci Technol. 2012;46(8):4458-4465.

49. Lacerda L, Russier J, Pastorin G, et al. Translocation mechanisms of chemically functionalised carbon nanotubes across plasma membranes. Biomaterials. 2012;33(11):3334-3343.
International Journal of Nanomedicine

\section{Publish your work in this journal}

The International Journal of Nanomedicine is an international, peerreviewed journal focusing on the application of nanotechnology in diagnostics, therapeutics, and drug delivery systems throughout the biomedical field. This journal is indexed on PubMed Central, MedLine, CAS, SciSearch $\AA$, Current Contents $₫ /$ Clinical Medicine,

\section{Dovepress}

Journal Citation Reports/Science Edition, EMBase, Scopus and the Elsevier Bibliographic databases. The manuscript management system is completely online and includes a very quick and fair peer-review system, which is all easy to use. Visit http://www.dovepress.com/ testimonials.php to read real quotes from published authors. 